\title{
Linear Confinement of a Scalar Particle Under Linear Central Potential Induced by the Effects of Violation of Lorentz Symmetry framework
}

\author{
Faizuddin Ahmed ${ }^{1}$ \\ National Academy Gauripur, Assam, 783331, India
}

\begin{abstract}
The relativistic quantum motion of a scalar particle under the effects of violation of the Lorentz symmetry in the presence of a linear confining potential is investigated. We see that the solution of the bound state to the modified Klein-Gordon equation can be obtained and a quantum effect characterized by the dependence of the magnetic field on the quantum numbers of the system is observed.
\end{abstract}

Keywords: Lorentz symmetry violation, Relativistic wave equations, non-electromagnetic potential, electric \& magnetic field, biconfluent Heun's function .

PACS Number(s): 03.65.Pm, 11.30.Cp, 11.30.Qc

\section{Introduction}

The Standard Model extension (SME) $[1,2,3,4,5,6,7,8,9,10]$ is the framework for investigating signals of Lorentz violation and studying properties of physical systems as it includes Lorentz violating terms in all sectors of the minimal Standard Model. The Lorentz-violating terms are generated as vacuum expectation values of tensors quantities, keeping the coordinate invariance of the extended theory [11].

\footnotetext{
${ }^{1}$ faizuddinahmed15@gmail.com ; faiz4U.enter@rediffmail.com
} 
In this paper, we study motion of a relativistic scalar particle under Lorentz symmetry breaking defined by a tensor $\left(K_{F}\right)_{\mu \nu \alpha \beta}$ out of the Standard Model Extension (SME) in the presence of a linear confining nonelectromagnetic potential. We analyze the effects of a linear central potential induced by the violation of the Lorentz symmetry and the solution of the bound state to the modified Klein-Gordon equation can be achieved. The relativistic scalar particle under the influence of non-electromagnetic potential has been of growing interest. Several authors have been studied the relativistic quantum systems in the presence of various kinds of electromagnetic and non-electromagnetic potential under the effects of Lorentz symmetry violation $[12,13,14,15,16,17,18,19]$. Noted that the gauge sector of Standard Model Extension consists of two classes that modifies the transport properties of space-time. These two classes are called the CPT-odd sector [1] and the CPT-even sector [20, 21, 22, 23, 24, 25].

The quantum dynamics of spin-0 scalar particle under the effects of Lorentz symmetry violation with a scalar potential $S(r)$ introduced via transformation $M \rightarrow M+S$ in the wave equation is given by $[1,2,12,13,14,15$, $16,17,18,19,22,23,25,26,27,28,29,30]$

$$
p^{\mu} p_{\mu} \Psi+\frac{\alpha}{4}\left(K_{F}\right)_{\mu \nu \alpha \beta} F^{\mu \nu}(x) F^{\alpha \beta}(x) \Psi=(M+S)^{2} \Psi
$$

where $\alpha$ is a constant, $F_{\mu \nu}(x)$ is the electromagnetic field tensor, $\left(K_{F}\right)_{\mu \nu \alpha \beta}$ is a dimensionless tensor that governs the Lorentz symmetry violation out of the Standard Model Extension. Note that linear confinement of a relativistic scalar particle with linear central potential induced by the Lorentz symmetry violation effects hasn't yet been investigated which is our aim in this paper.

\section{Relativistic Scalar particle under LSV ef- fects with a Linear Confining Potential}

The Klein-Gordon equation under the effects of Lorentz symmetry violation defined by the Eq. (1) in the background of the Minkowski space-time is 
given by

$$
\begin{aligned}
& {\left[-\frac{\partial^{2}}{\partial t^{2}}+\frac{\partial^{2}}{\partial r^{2}}+\frac{1}{r} \frac{\partial}{\partial r}+\frac{\partial^{2}}{\partial z^{2}}+\frac{1}{r^{2}} \frac{\partial^{2}}{\partial \phi^{2}}\right] \Psi+\frac{\alpha}{4}\left(K_{F}\right)_{\mu \nu \alpha \beta} F^{\mu \nu}(x) F^{\alpha \beta}(x) \Psi} \\
& =(M+S)^{2} \Psi .
\end{aligned}
$$

Using the properties of the tensor $\left(K_{F}\right)_{\mu \nu \alpha \beta}[1,3,4,8,22,23,25,12,13$, $14,26,27$, we can rewrite (2) in the following form :

$$
\begin{aligned}
& {\left[-\frac{\partial^{2}}{\partial t^{2}}+\frac{\partial^{2}}{\partial r^{2}}+\frac{1}{r} \frac{\partial}{\partial r}+\frac{1}{r^{2}} \frac{\partial^{2}}{\partial \phi^{2}}+\frac{\partial^{2}}{\partial z^{2}}\right] \Psi} \\
& +\left[-\frac{\alpha}{2}\left(\kappa_{D E}\right)_{i j} E^{i} E^{j}+\frac{\alpha}{2}\left(\kappa_{H B}\right)_{j k} B^{i} B^{j}-\alpha\left(\kappa_{D B}\right)_{j k} E^{i} B^{j}\right] \Psi \\
& =(M+S)^{2} \Psi .
\end{aligned}
$$

We choose the scenario of the Lorentz symmetry violation determined by the non-null components $\left(\kappa_{D E}\right)_{11}=$ const $=\kappa_{1},\left(\kappa_{H B}\right)_{33}=$ const $=\kappa_{2}$ and $\left(\kappa_{D B}\right)_{13}=$ const $=\kappa_{3}$ with the following field configuration $[12,13,14,26$, $27,15,16,28,29,30]$ :

$$
\vec{B}=B_{0} \hat{z} \quad, \quad \vec{E}=\frac{\lambda}{2} r \hat{r}
$$

where $B_{0}>0, \hat{z}$ is a unit vector in the $z$-direction, $\lambda$ is a constant associated with a linear charge density of electric charges along the axial direction, and $\hat{r}$ is the unit vector in the radial direction.

Hence, equation (3) using the configuration (4) becomes

$$
\begin{aligned}
& {\left[-\frac{\partial^{2}}{\partial t^{2}}+\frac{\partial^{2}}{\partial r^{2}}+\frac{1}{r} \frac{\partial}{\partial r}+\frac{1}{r^{2}} \frac{\partial^{2}}{\partial \phi^{2}}+\frac{\partial^{2}}{\partial z^{2}}\right] \Psi} \\
& +\left[-\frac{\alpha \kappa_{1} \lambda^{2}}{8} r^{2}+\frac{\alpha \kappa_{2} B_{0}^{2}}{2}-\frac{\alpha \lambda B_{0} \kappa_{3}}{2} r\right] \Psi=(M+S)^{2} \Psi
\end{aligned}
$$

Since the metric is independent of $(t, \phi, z)$, let the solution to the Eq. (6) is

$$
\Psi(t, r, \phi, z)=e^{i(-E t+l \phi+k z)} \psi(r)
$$

where $E, l$ have their usual meaning, and $k$ is a constant. 
In this work, we are mainly interested in a linear confining potential $S(r)=\eta_{L} r$. This type of potential has been used in the relativistic quantum mechanics. Therefore, using the linear confining potential into the Eq. (5) and using the solution (6), we obtain the following radial wave equation for $\psi(r)$ :

$$
\psi^{\prime \prime}(r)+\frac{1}{r} \psi^{\prime}(r)+\left[\Sigma-\frac{l^{2}}{r^{2}}-\omega^{2} r^{2}-\beta r\right] \psi(r)=0,
$$

where

$$
\begin{aligned}
\Sigma & =E^{2}-M^{2}-k^{2}+\frac{1}{2} \alpha \kappa_{2} B_{0}^{2}, \\
\omega & =\sqrt{\frac{1}{8} \alpha \kappa_{1} \lambda^{2}+\eta_{L}^{2}}, \\
\beta & =2 M \eta_{L}+\frac{1}{2} \alpha \lambda B_{0} \kappa_{3} .
\end{aligned}
$$

Transforming $\xi=\sqrt{\omega} r$ in the above equation (7), we have

$$
\left[\frac{d^{2}}{d \xi^{2}}+\frac{1}{\xi} \frac{d}{d \xi}+\zeta-\xi^{2}-\frac{l^{2}}{\xi^{2}}-\theta \xi\right] \psi(\xi)=0,
$$

where

$$
\zeta=\frac{\Sigma}{\omega} \quad, \quad \theta=\frac{\beta}{\omega^{\frac{3}{2}}} .
$$

Now we impose the requirement that the wave-function $\psi(x)$ is finite both at the origin $x \rightarrow 0$ and at $x \rightarrow \infty$. Suppose the possible solution to the Eq. (9) is

$$
\psi(\xi)=\xi^{|l|} e^{-\frac{1}{2}(\xi+\theta) \xi} H(\xi) .
$$

Substituting the solution (11) into the Eq. (9), we obtain the following equation

$$
H^{\prime \prime}(\xi)+\left[\frac{1+2|l|}{\xi}-2 \xi-\theta\right] H^{\prime}(\xi)+\left[-\frac{\frac{\theta}{2}(1+2|l|)}{\xi}+\Theta\right] H(\xi)=0,
$$

where

$$
\Theta=\zeta+\frac{\theta^{2}}{4}-2(1+|l|) .
$$


Equation (12) is the biconfluent Heun's differential equation [15, 31, 32] with $H(\xi)$ is the Heun polynomials function.

The above equation (12) can be solved by the Frobenius method. Writing the solution as a power series expansion around the origin [33]:

$$
H(\xi)=\sum_{i=0}^{\infty} d_{i} \xi^{i}
$$

Substituting the power series solution into the Eq. (14), we obtain the following recurrence relation

$$
d_{n+2}=\frac{1}{(n+2)(n+2+2|l|)}\left[\left\{\eta+\theta\left(n+\frac{3}{2}+|l|\right)\right\} d_{n+1}-(\Theta-2 n) d_{n}\right] .
$$

With the few coefficients are

$$
\begin{aligned}
& d_{1}=\frac{\theta}{2} d_{0}, \\
& d_{2}=\frac{1}{4(1+|l|)}\left[\theta\left(|l|+\frac{3}{2}\right) d_{1}-\Theta d_{0}\right] .
\end{aligned}
$$

The power series expansion $H(\xi)$ becomes a polynomial of degree $n$ by imposing the following two conditions $[12,13,14,15]$

$$
\begin{aligned}
\Theta & =2 n, \quad(n=1,2, \ldots) \\
d_{n+1} & =0 .
\end{aligned}
$$

By analyzing the first condition, we obtain following equation of the energy eigenvalue $E_{n, l}$ :

$$
E_{n, l}= \pm \sqrt{M^{2}+k^{2}+2 \omega(n+1+|l|)-\frac{1}{2} \alpha B_{0}^{2} \kappa_{2}-\frac{\beta^{2}}{4 \omega^{2}}}
$$

It is worth mentioning that we have adjusted the parameters such that $\left(M^{2}+k^{2}+2 \omega(n+1+|l|)\right)>\left(\frac{1}{2} \alpha B_{0}^{2} \kappa_{2}+\frac{\beta^{2}}{4 \omega^{2}}\right)$ and thus, the energy eigenvalue is real. Note that the Eq. (18) is not the general expression of energy eigenvalue of a relativistic scalar particle. One can obtain the individual 
energy level and the eigenfunction one by one by imposing the additional recurrence condition $d_{n+1}=0$ on the eigenvalue problem.

The corresponding wave-functions are given by

$$
\psi_{n, l}(\xi)=\xi^{|l|} e^{-\frac{1}{2}\left[\xi+\frac{\beta}{\omega^{\frac{3}{2}}}\right] \xi} H(\xi) .
$$

Now, we evaluate the individual energy levels and the eigenfunctions one by one as done in [15]. For example, $n=1$, we have $\Theta=2$ and $d_{2}=0$ which implies

$$
\begin{aligned}
& \Rightarrow \frac{2}{\theta\left(\frac{3}{2}+|l|\right)} d_{0}=\frac{\theta}{2} d_{0} \\
& \Rightarrow \theta^{2}=\frac{4}{\frac{3}{2}+|l|} \Rightarrow \frac{\beta^{2}}{\omega^{3}}=\frac{4}{\frac{3}{2}+|l|} \Rightarrow \beta_{1, l}=\sqrt{\frac{4 \omega^{3}}{\frac{3}{2}+|l|}}
\end{aligned}
$$

a constraint on the parameter $\beta_{1, l}$, that is, on the magnetic field. We can see from Eq. (20), that the allowed values of the magnetic field depends on the quantum numbers of the system $\{n, l\}$, the parameters associated with the background of the Lorentz Symmetry Violation, and the potential parameters.

The allowed values of the magnetic field for the radial mode $n=1$ is

$$
B_{0}^{1, l}=\frac{\left(\beta_{1, l}-2 M \eta_{L}\right)}{\frac{1}{2} \alpha \lambda \kappa_{3}}
$$

Therefore, the ground state energy level for the radial mode $n=1$ is given by

$E_{1, l}= \pm \sqrt{M^{2}+k^{2}+\sqrt{\frac{1}{2} \alpha \kappa_{1} \lambda^{2}+4 \eta_{L}^{2}}\left\{(2+|l|)-\frac{1}{(3+2|l|)}\right\}-\frac{1}{2} \alpha\left(B_{0}^{1, l}\right)^{2} \kappa_{2} .}$

And the ground state eigenfunction is

$$
\psi_{1, l}(\xi)=\xi^{\sqrt{l^{2}+\eta_{c}^{2}}} e^{-\frac{1}{2}\left(\xi+2 d_{1}\right) \xi}\left(1+d_{1} \xi\right),
$$


where we have chosen $d_{0}=1$ and

$$
d_{1}=\frac{1}{\sqrt{\frac{3}{2}+|l|}} .
$$

We can see that the lowest energy state (22) plus the ground state wave function (23)-(24) is defined for the radial mode $n=1$, instead of $n=0$. This effect arises due to the presence of linear confining potential and the Lorentz symmetry violation in the relativistic quantum system.

\section{Conclusions}

We have investigated the effects of a linear central potential induced by the violation of the Lorentz symmetry background on a relativistic scalar particle under a linear confining potential. After solving the wave equation, we have obtained the non-compact expression of the energy eigenvalues (18) and the wave function (19). By imposing the recurrence condition $d_{n+1}=0$, one can obtain the individual energy levels and the wave function, for example, the lowest state energy level (21) and the corresponding wave function (22)-(23) with the restriction on the magnetic field (20) for the radial mode $n=1$. We have seen a quantum effect due to the dependence of the magnetic field $B_{0}$ on the quantum numbers $\{n, l\}$ of the system as well as on the Lorentz symmetry breaking parameters.

\section{Conflict of Interest}

There is no conflict of interest regarding publication this paper.

\section{Data Availability}

No data has been used to prepare this paper. 


\section{References}

[1] D. Colladay and V. A. Kostelecky, Phys. Rev. D 55, 6760 (1997).

[2] D. Colladay and V. A. Kostelecky, Phys. Rev. D 58, 116002 (1998).

[3] S. R. Coleman and S. L. Glashow, Phys. Rev. D 59, 116008 (1999).

[4] V. A. Kostelecky and S. Samuel, Phys. Rev. Lett. 63, 224 (1989).

[5] V. A. Kostelecky and S. Samuel, Phys. Rev. Lett. 66, 1811 (1991).

[6] V. A. Kostelecky and S. Samuel, Phys. Rev. D 39, 683 (1989).

[7] V. A. Kostelecky and S. Samuel, Phys. Rev. D 40, 1886 (1989).

[8] V. A. Kostelecky and R. Potting, Nucl. Phys. B 359, 545 (1991).

[9] V. A. Kostelecky and R. Potting, Phys. Lett. B 381, 89 (1996).

[10] V. A. Kostelecky and R. Potting, Phys. Rev. D 51, 3923 (1995).

[11] V. A. Kostelecky and R. Lehnert, Phys. Rev. D 63, 065008 (2001).

[12] K. Bakke and H. Belich, Ann. Phys. (N. Y.) 354, 1 (2015).

[13] K. Bakke and H. Belich, Ann. Phys. (N. Y.) 373, 115 (2016).

[14] R. L. L. Vitoria and H. Belich, Eur. Phys. J. C 78, 999 (2018).

[15] F. Ahmed, IJMPA (2021), DOI:10.1142/S0217751X21501281.

[16] R. L. L. Vitoria, H. Belich and K. Bakke, Adv. High Energy Phys. 2017, 6893084 (2017).

[17] K. Bakke and H. Belich, Ann. Phys. (N. Y.) 360, 596 (2015).

[18] R. L. L. Vitoria, K. Bakke and H. Belich, Ann. Phys. (N. Y.) 399, 117 (2018). 
[19] R. L. L. Vitoria, H. Belich and K. Bakke, Eur. Phys. J. Plus 132, 25 (2017).

[20] A. P. Baeta Scarpelli, H. Belich, J. L. Boldo, L. P. Colatto, J. A. HelayelNeto, and A. L. M. A. Nogueira, Nucl. Phys. B (Proc. Suppl.) 127, 105 (2004).

[21] S. Carroll, G. Field and R. Jackiw, Phys. Rev. D 41, 1231 (1990).

[22] V. A. Kostelecky and M. Mewes, Phys. Rev. Lett. 87, 251304 (2001).

[23] V. A. Kostelecky and M. Mewes, Phys. Rev. D 66, 056005 (2002).

[24] V. A. Kostelecky and M. Mewes, Phys. Rev. Lett. 97, 140401 (2006).

[25] H. Belich, F. J. L. Leal, H. L. C. Louzada and M. T. D. Orlando, Phys. Rev. D 86, 125037 (2012).

[26] M. Ericsson and E. Sjoqvist, Phys. Rev. A 65, 013607 (2001).

[27] I. C. Fonseca and K. Bakke, J. Math. Phys. 56, 062107 (2015).

[28] F. Ahmed, DOI : 10.20944/preprints202105.0782.v1.

[29] F. Ahmed, DOI : 10.20944/preprints202106.0003.v1.

[30] F. Ahmed, DOI : 10.20944/preprints202106.0006.v1.

[31] A. Ronveaux, Heun's Differential Equations, Oxford University Press, Oxford ( 1995).

[32] S. Y. Slavyanov and W. Lay, Special Functions: A Unified Theory Based in Singularities, Oxford University Press, New York (2000).

[33] G. B. Arfken and H. J. Weber, Mathematical Methods For Physicists, Elsevier Academic Press, London (2005). 